\title{
BALAYAGE BY FOURIER TRANSFORMS WTTH SPARSE FREQUENCIES IN COMPACT ABELIAN TORSION GROUPS
}

\author{
GEORGE S. SHAPIRO
}

\begin{abstract}
Let $\Lambda$ be a discrete subset of a LCA group and $E$ a compact subset of the dual group $\Gamma$. Balayage is said to be possible for $(\Lambda, E)$ if the Fourier transform of each measure on $G$ is equal on $E$ to the Fourier transform of some measure supported by $\Lambda$.

For a class of infinite compact metrizable $\Gamma$, including all such torsion groups, we show how to construct $E \subset \Gamma$ such that there are arbitrarily sparse sets $\Lambda$ with balayage possible for $(\Lambda, E) . E$ is, moreover, large enough that the set of products $E \cdot E \cdot E=\Gamma$.
\end{abstract}

Let $G$ be a locally compact abelian group with dual group $\Gamma$, both written multiplicatively. For $\mu \in M(G)$, we denote by $\hat{\mu}$ the inverse Fourier transform $\hat{\mu}(\gamma)=\int_{G}\langle\gamma, x\rangle d \mu(x)$.

We recall a notion introduced by Beurling [1].

Definition 1. If $\Lambda \subset G$ is discrete and $E \subset \Gamma$ is compact, then balayage is said to be possible for $(\Lambda, E)$ if, for every $\mu \in M(G)$ there is some $\nu \in M(\Lambda)$ with $\hat{\nu}(\gamma)=\hat{\mu}(\gamma)$ for all $\gamma \in E$.

In [3, p. 151] and [4, p. 160], Kahane shows the existence of fairly large sets $E$ (of Cantor type) in the circle group $T$ with the following property. Given any sequence $\left\{\phi_{n}\right\}_{1}^{\infty}$ of real-valued functions ( $\phi_{n}$ having $n-1$ arguments) there is a set $\Lambda=\left\{\lambda_{n}\right\}_{1}^{\infty} \subset Z$ with balayage possible for $(\Lambda, E)$ and with $\lambda_{n} \geqslant \phi_{n}\left(\lambda_{1}, \lambda_{2}, \ldots, \lambda_{n-1}\right)$ for all $n$. (Kahane does not use the terminology of balayage.) In [5], similar examples of such sets $E$ were constructed, in the real line, using a different technique. Such sets were called BAS sets (for "balayage with arbitrarily sparse frequencies"). The purpose of this paper is to define the notion of BAS sets for general LCA groups, and to give, for metrizable compact torsion groups, fairly "large" examples of such sets. The technique used here involves a necessary modification of that of [5].

Definition 2. $E \subset \Gamma$ is called a BAS set if, given any sequence of functions, $\left\{F_{n}\right\}_{1}^{\infty}$, where $F_{n}$ takes values in the collection of compact subsets of $G$, there is a set $\Lambda=\left\{\lambda_{n}\right\}_{1}^{\infty}$ with balayage possible for $(\Lambda, E)$ and with

$$
\lambda_{n} \notin F_{n}\left(\lambda_{1}, \lambda_{2}, \ldots, \lambda_{n-1}\right)
$$

Received by the editors October 27, 1977.

AMS (MOS) subject classifications (1970). Primary 43A25; Secondary 42A44, 43A70.

Key words and phrases. Balayage in Fourier transforms, arbitrarily sparse frequencies, compact abelian torsion group, approximation by characters. 
for all $n$. (Here, $F_{1}$ is just a compact subset of $G$.)

Let $B(E)$ be the Banach algebra of restrictions to $E$ of Fourier transforms of measures in $M(G)$ normed by

$$
\|\phi\| \widehat{B(E)}=\inf \{\|\mu\|: \mu \in M(G) \text { and } \hat{\mu} \mid E=\phi\} .
$$

It is straightforward to modify [5, Theorem 6.1], which was stated only for the real case, to prove

THEOREM 1. If there is some $\delta<1$ and a sequence $\left\{t_{n}\right\}_{1}^{\infty} \subset G$ with $\lim _{n \rightarrow \infty} t_{n}$ $=\infty$ and with

$$
\left\|1-\left\langle\cdot, t_{n}\right\rangle\right\|_{B(E)}<\delta
$$

for all $n$, then $E$ is a BAS set. (Here, $\langle\cdot, x\rangle$ is the function $\gamma \mapsto\langle\gamma, x\rangle$.)

Theorem 1 suffices for constructing examples of fairly large BAS sets in many metrizable groups (for example, the groups of $p$-adic integers). Suppose, however, that every element of $\Gamma$ has order at most 6 . Then, for $x \in G$ and $\gamma \in \Gamma$, if $\langle\gamma, x\rangle \neq 1$, then $\langle\gamma, x\rangle$ is a nontrivial $n$th root of 1 for some $n \leqslant 6$ and, thus,

$$
|1-\langle\gamma, x\rangle| \geqslant 1 \text {. }
$$

It is, therefore, impossible for the hypotheses of Theorem 1 to apply to $E$ unless $\left\langle\gamma, t_{n}\right\rangle=1$ for all $\gamma \in E$. In such a case, $E$ must be quite thin. (For example, no finite product $E \cdot E \cdot \ldots \cdot E$ can have interior.) Via a somewhat different route, however, we can prove

THEOREM 2. If $\Gamma$ is an infinite, metrizable, compact, abelian, torsion group, then $\Gamma$ contains a $B A S$ set $E$ with $E \cdot E \cdot E=\Gamma$.

Proof. By $[2,25.9]$, we may write $\Gamma$ as a product

$$
\Gamma=\stackrel{\infty}{n=1}_{n}^{\infty} \Gamma_{n}
$$

where each $\Gamma_{n}$ is a nontrivial, finite group (and $\Gamma$ has the product topology). Then $G$ is a discrete direct sum

$$
G=\bigoplus_{n=1}^{\infty} G_{n}
$$

where each $G_{n}$ is the dual group of $\Gamma_{n}$ (and so is isomorphic to $\Gamma_{n}$ ). Here typical elements $x$ and $\gamma$ of $G$ and $\Gamma$ respectively are sequences

$$
x=\langle x(1), x(2), x(3), \ldots\rangle \text { and } \gamma=\langle\gamma(1), \gamma(2), \gamma(3), \ldots\rangle
$$

where each $x(n) \in G_{n}$ and $\gamma(n) \in \Gamma_{n}$ and all but finitely many of the $x(n)$ equal 1 . The duality between $G$ and $\Gamma$ is given by

$$
\langle\gamma, x\rangle=\prod_{1}^{\infty}\langle\gamma(n), x(n)\rangle
$$

where all but finitely many terms in the product are 1 .

$$
\text { For } r=1,2,3 \text {, let }
$$




$$
I_{r}=\{n \geqslant 1: n \equiv r(\bmod 3)\}
$$

and let

$$
E_{r}=\left\{\gamma \in \Gamma: \gamma(n)=1 \text { if } n \notin I_{r}\right\} .
$$

Finally, we let $E=E_{1} \cup E_{2} \cup E_{3}$. We note that it is clear that $E \cdot E \cdot E=$ $\Gamma$.

To verify that $E$ is a BAS set requires some auxiliary constructions which show the possibility of approximating the constant function 1 on $E$ by a linear combination of characters (instead of just by characters as in Theorem 1).

$s_{m}$ will denote an element of $G$ such that

$s_{m}(n)=1$ if $n \neq m$ and

$s_{m}(m) \neq 1$.

(The exact choice of $s_{m}(m)$ is irrelevant. What we need is that the various $s_{m}$ be distinct.)

$\delta(x)$ denotes the unit point mass at $x$.

We shall use the letters $i, j$ and $k$ (subscripted or not) to denote elements of $I_{1}, I_{2}$ and $I_{3}$ respectively. With this convention in mind, we define

$$
\mu=\mu(i, j, k)=\frac{1}{3}\left(\delta\left(s_{i}\right)+\delta\left(s_{j}\right)+\delta\left(s_{k}\right)\right)
$$

and

$$
\nu=\nu(i, j, k)=\frac{1}{3}\left(\delta(0)-\delta\left(s_{i} s_{j} s_{k}\right)\right) .
$$

We note that if $\gamma \in E$ then at most one of $\left\langle\gamma, s_{i}\right\rangle,\left\langle\gamma, s_{j}\right\rangle$ and $\left\langle\gamma, s_{k}\right\rangle$ can fail to equal 1. Thus, for $\gamma \in E$,

$$
\begin{aligned}
1-\hat{\mu}(\gamma) & =1-\frac{1}{3}\left(\left\langle\gamma, s_{i}\right\rangle+\left\langle\gamma, s_{j}\right\rangle+\left\langle\gamma, s_{k}\right\rangle\right) \\
& =\frac{1}{3}-\frac{1}{3}\left\langle\gamma, s_{i}\right\rangle\left\langle\gamma, s_{j}\right\rangle\left\langle\gamma, s_{k}\right\rangle=\hat{\nu}(\gamma) .
\end{aligned}
$$

Since $\|\nu(i, j, k)\|=\frac{2}{3}$ we conclude that

$$
\left\|1-\mu(i, j, k)^{\wedge}\right\|_{B(E)} \leqslant \frac{2}{3} .
$$

Enumerate $G=\left\{g_{n}\right\}_{1}^{\infty}$.

Suppose functions $\left\{F_{n}\right\}_{1}^{\infty}$ as in Definition 2 are given. We construct $\Lambda$ inductively, adding three elements to $\Lambda$ for each element of $G$. Note that $S x^{-1}$ denotes $\left\{s x^{-1}: s \in S\right\}$. The fact that each $F_{n}$ takes values in the collection of finite subsets of $G$ should be borne in mind.

Pick $i_{1} \in I_{1}$ such that $s_{i_{1}} \notin F_{1} g_{1}^{-1}$ and set $\lambda_{1}=s_{i_{1}} g_{1}$.

Pick $j_{1} \in I_{2}$ such that $s_{j_{1}} \notin F_{2}\left(\lambda_{1}\right) g_{1}^{-1}$ and set $\lambda_{2}=s_{j_{1}} g_{1}$.

Pick $k_{1} \in I_{3}$ such that $s_{k_{1}} \notin F_{3}\left(\lambda_{1}, \lambda_{2}\right) g_{1}^{-1}$ and set $\lambda_{3}=s_{k_{1}} g_{1}$.

Pick $i_{2} \in I_{2}$ such that $s_{i_{2}} \notin F_{4}\left(\lambda_{1}, \lambda_{2}, \lambda_{3}\right) g_{2}^{-1}$ and set $\lambda_{4}=s_{i_{2}} g_{2}$ and continue in this manner.

Let $\Lambda=\left\{\lambda_{n}\right\}_{1}^{\infty}$. Clearly, $\Lambda$ satisfies (1). We must show that balayage is possible for $(\Lambda, E)$. To this end, let $B_{\Lambda}(E)=\{\hat{\beta} \mid E: \beta \in M(\Lambda)\} \subset B(E)$. $B_{\Lambda}(E)$ becomes a Banach space under the norm 


$$
\|\psi\|_{B_{\Lambda}(E)}=\inf \{\|\beta\|: \beta \in M(\Lambda) \text { and } \hat{\beta} \mid E=\psi\}
$$

It clearly suffices to show that $B_{\Lambda}(E)=B(E)$.

Suppose $\phi \in B(E)$. Then there is some $\alpha=\sum a_{n} \delta\left(g_{n}\right) \in M(G)$ with $\hat{\alpha} \mid E$ $=\phi$ and with $\Sigma\left|a_{n}\right|=\|\alpha\| \leqslant \frac{9}{8}\|\phi\|_{B(E)}$.

Let

$$
\beta=\sum a_{n} \delta\left(g_{n}\right) * \mu\left(i_{n}, j_{n}, k_{n}\right)
$$

Then, $\beta \in M(\Lambda)$ because $\delta\left(g_{n}\right) * \mu\left(i_{n}, j_{n}, k_{n}\right)$ is supported by $\left\{s_{i_{n}} g_{n}, s_{j_{n}} g_{n}, s_{k_{n}} g_{n}\right\}=\left\{\lambda_{3 n-2}, \lambda_{3 n-1}, \lambda_{3 n}\right\}$. Set $\psi=\hat{\beta} \mid E$. Then $\psi \in B_{\Lambda}(E)$. Noting that $\left\|\mu\left(i_{n}, j_{n}, k_{n}\right)\right\|=1$, we see that

$$
\|\psi\|_{B_{\Lambda}(E)} \leqslant \sum\left|a_{n}\right| \leqslant \frac{9}{8}\|\phi\|_{B(E)} .
$$

We have also

$$
\begin{aligned}
\|\phi-\psi\|_{B(E)} & =\left\|\sum a_{n}\left\langle\cdot, g_{n}\right\rangle\left(1-\mu\left(i_{n}, j_{n}, k_{n}\right)\right)^{\wedge}\right\|_{B(E)} \\
& \leqslant \frac{2}{3} \sum\left|a_{n}\right| \leqslant \frac{3}{4}\|\phi\|_{B(E)}
\end{aligned}
$$

where we have used (2) and the fact that $B(E)$ is a Banach algebra. A standard iteration now shows that $\phi \in B_{\Lambda}(E)$ and, indeed,

$$
\|\phi\|_{B_{\Lambda}(E)} \leqslant \frac{9}{2}\|\phi\|_{B(E)}
$$

and the theorem follows. (The point is that $\frac{9}{2}=\frac{9}{8} \sum_{0}^{\infty}\left(\frac{3}{4}\right)^{n}$.)

REMARKS. 1. Replacing $\frac{9}{8}$ by $1+\varepsilon$ for $\varepsilon>0$ shows that we can replace the factor $\frac{9}{2}$ (in (3)) by 3 .

2. The same result and proof hold in the more general case where the various $\Gamma_{n}$ are merely compact and metrizable (the latter being required to make $G$ countable).

\section{BIBLIOGRAPHY}

1. A. Beurling, On balayage of measures in Fourier transforms, Notes from a seminar at the Institute for Advanced Study, Princeton, N. J., 1959-60 (unpublished).

2. E. Hewitt and K. A. Ross, Abstract harmonic analysis. I, Grundlehren Math. Wiss., Band 115, Springer-Verlag, Berlin and New York, 1963.

3. J.-P. Kahane, Séries de Fourier absolument convergentes, Ergebnisse der Mathematik und ihrer Grenzgebiete, Band 50, Springer-Verlag, Berlin and New York, 1970.

4. J.-P. Kahane and R. Salem, Ensembles parfaits et séries trigonométriques, Actualités Sci. Indust., No. 1301, Hermann, Paris, 1963.

5. G. S. Shapiro, Balayage in Fourier transforms: general results, perturbation and balayage with sparse frequencies, Trans. Amer. Math. Soc. 225 (1977), 183-198.

Department of Mathematics, Brooklyn College (CUNY), Brookiyn, New York 11210 